\title{
Crohn's Disease Following Rituximab Treatment for Follicular Lymphoma in a Patient with Synchronous Gastric Signet Ring Cells Carcinoma: A Case Report and Literature Review
}

\author{
Elisabetta Cavalcanti, $\mathrm{PhD}$ \\ Raffaele Armentano, MD \\ Ivan Lolli, MD²
}

${ }^{1}$ Histopathology Unit of National Institute of Gastroenterology "S. de Bellis" and ${ }^{2}$ Oncology Unit of National Institute of Gastroenterology "S. de Bellis", Research Hospital, Castellana Grotte, Bari, Italy
Recently, there have been a few reports of rituximab (RTX)-induced Crohn's disease, but there is no literature available on successful long-term treatment and the clinical outcome of this condition. We retrospectively analyzed the clinical data of a rare case of Crohn's disease induced by RTX administered as induction and prolonged maintenance therapy of a follicular lymphoma, diagnosed synchronously with a gastric signet ring cells carcinoma, treated at our hospital.

\section{Key words}

Rituximab, Crohn's disease, Lymphoma, Gastric signet ring cells carcinoma

Correspondence: Elisabetta Cavalcanti, $\mathrm{PhD}$ Histopathology Unit of National Institute of Gastroenterology "S. de Bellis,"

Research Hospital, Via Turi 27,

70013 Castellana Grotte (Ba), Italy

Tel: 39-0804994111

Fax: 39-0804994340

E-mail: elisabetta.cavalcanti@irccsdebellis.it

Received April 30, 2020

Accepted July 10, 2020

Published Online July 13, 2020

\section{Introduction}

Crohn's disease is an inflammatory bowel disease (IBD) inducing abdominal pain, severe diarrhea, fatigue, weight loss, and malnutrition. Signs and symptoms of Crohn's disease range from mild to severe, with periods of disease remission. The most common areas affected by Crohn's disease are the last part of the small intestine and the colon but any segment of the gastrointestinal tract may be affected. The pathogenesis is complex, stemming from genetic susceptibility and mucosal immunity dysfunction as a result of B-cell depletion with a regulatory function to control inflammation [1].

Rituximab (RTX) is an IgG1, anti-CD20 chimeric monoclonal antibody that induces a selective transient depletion of peripheral CD20-positive B cells [2]. RTX is part of the standard treatment of patients with B-cell non-Hodgkin's lymphoma (NHL), including follicular lymphoma (FL). The mechanisms of action are not fully clarified. A number of antitumor effects have been suggested, including antibody-dependent cellular cytotoxicity, complement-dependent cytotoxicity, the induction of apoptosis and sensitization of B cells to chemotherapy [3]. RTX is usually well-tolerated but that adverse events can occur, such as severe mucocutaneous reactions, infusional reactions, progressive multifocal leukoencephalopathy, acute respiratory distress syndrome and cardiovascular events. Recently, RTX has also been associated with adverse gastrointestinal effects, including diarrhea and bowel perforation, and recent reports have associated RTX with the development of de novo IBD [4,5].

Gastrointestinal toxicities are uncommon, indeed, only two reports of RTX-induced Crohn's disease have been published in the literature [6,7]. FL is an indolent B-cell lymphoproliferative disorder of transformed follicular center B cells. It is the second most common subtype of NHL diagnosed in Western countries. The neoplastic cells consist of a mixture of centrocytes (small to medium-sized cells) and centroblasts (large cells). The clinical aggressiveness of the tumor increases with increasing numbers of centroblasts [8]. Gastric signet 

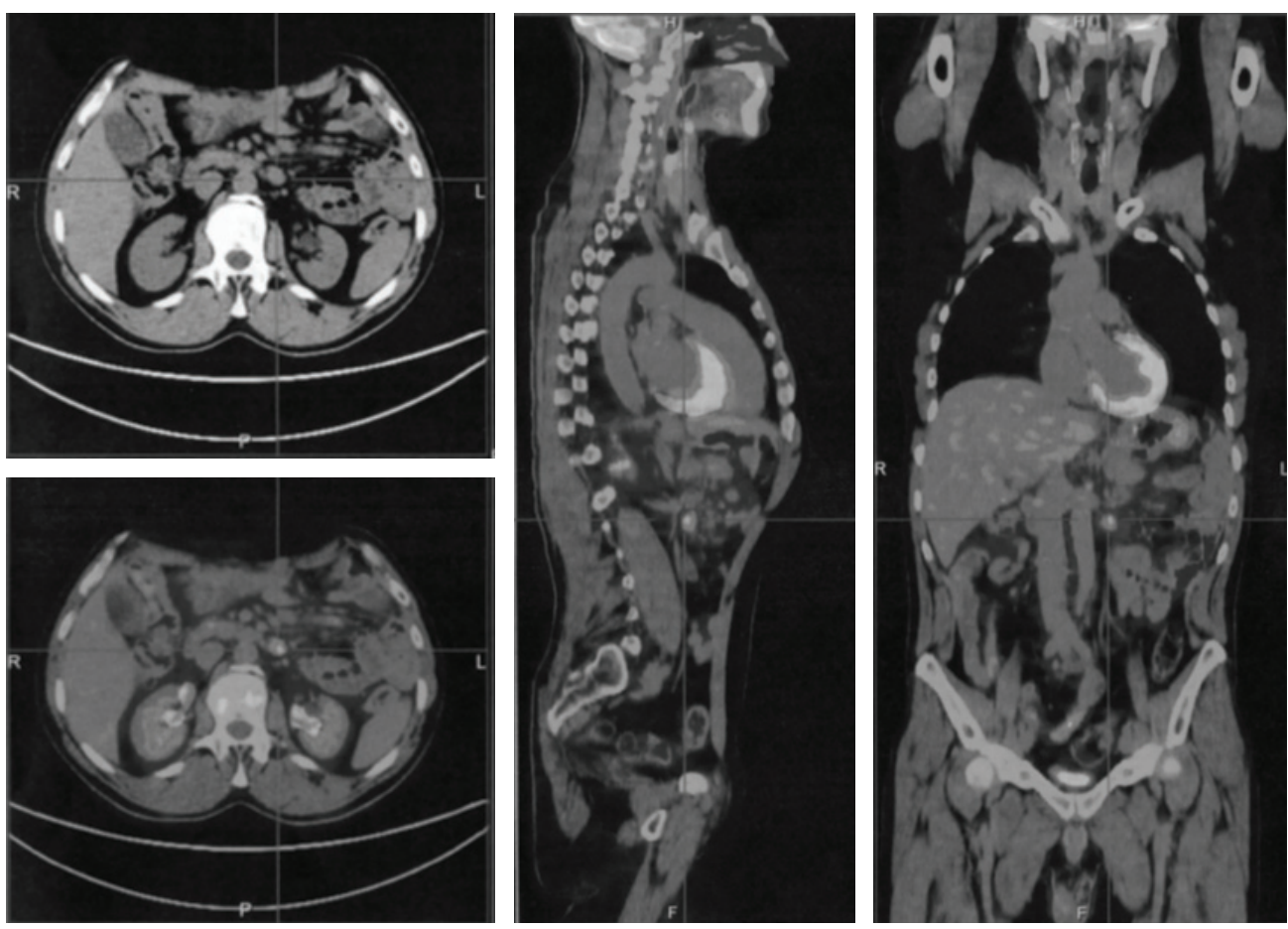

Fig. 1. Positron emission tomography scan showing massive ${ }^{18} \mathrm{~F}$-fluorodeoxyglucose uptake (maximum standardized uptake value, 7 ) and mesenteric lymph nodes enlargement.

ring cells carcinoma (SRCC) is defined as an adenocarcinoma in which the majority of cells (>50\%) consists of isolated or small groups of malignant non-cohesive cells containing intracytoplasmic mucin. Surgical resection with lymphadenectomy is the treatment of choice for gastric signet ring cell (SRC). To date, there has been no evaluation of the sensitivity of gastric SRCC toward chemotherapeutic drugs [9]. Synchronous FL and gastric SRC adenocarcinoma are extremely rare. Here, we present a case of prolonged RTX maintenance treatment-induced Crohn's disease in a patient with synchronous FL and gastric SRC adenocarcinoma.

\section{Case Report}

A 48-year-old male was admitted to our hospital in July 2009 due to upper abdominal pain, nausea, vomiting, and weight loss lasting 4 weeks. The patient had no personal or family medical history of a malignant neoplasm. Gastroscopy revealed an antropyloric neoformation, $3 \mathrm{~cm}$ in diameter, and biopsy of the gastric lesion was positive for gastric SRC carcinoma. Computed tomography scan confirmed the gastric tumor and showed a coexistent massive mesenteric abdominal mass, with enlarged para-aortic, aorto-caval, and coeliac axis lymph nodes. A subtotal gastrectomy with D2 lympho-adenectomy and an excisional mesenteric node biopsy were performed. Histologic examination was consist- ent with two synchronous malignancies: a poorly differentiated intramucosal gastric SRC adenocarcinoma with embolic micrometastases in $2+/ 19$ nodes of the stomach greater curvature (pT1,N1, M0, stage IB), and a follicular NHL (FL), grade3a ( $>15$ centroblasts/high-power field and centrocytes present in the sample). Immunohistochemical staining of B FL cells revealed the co-expression of CD20, BCL6, BCL2, and CD79a within the B neoplastic follicles and a Ki-67 index $>20 \%$. Bone marrow biopsy showed sporadic interstitial aggregates of small lymphoid CD20 and CD3 positive elements. After surgery, the patient showed a good recovery and was discharged on postoperative day 9 . The surgical procedure was considered appropriate for early-stage I-B gastric cancer so no adjuvant chemotherapy was administered. However, a systemic chemotherapeutic regimen was selected for the FL bulky disease. The patient received seven cycles of a chemotherapy regimen including, on day 1: cyclophosphamide 750 $\mathrm{mg} / \mathrm{m}^{2}$, doxorubicin $50 \mathrm{mg} / \mathrm{m}^{2}$, and vincristine $1.4 \mathrm{mg} / \mathrm{m}^{2}$, and on days 1-5: prednisone $100 \mathrm{mg}$ (CHOP regimen).The treatment was well-tolerated and induced a complete response. Two years later, a positron emission tomography (PET) scan showed disease recurrence, with mesenteric lymph nodes enlargement and increased ${ }^{18} \mathrm{~F}$-fluorodeoxyglucose uptake (maximum standardized uptake value, 7) (Fig. 1). The patient was treated with eight cycles of R-CNOP (day 1: RTX $375 \mathrm{mg} / \mathrm{m}^{2}$; day 2: cyclophosphamide $750 \mathrm{mg} /$ $\mathrm{m}^{2}$, mitoxantrone $10 \mathrm{mg} / \mathrm{m}^{2}$, vincristine $2 \mathrm{mg}$; days 2-6: pre- 

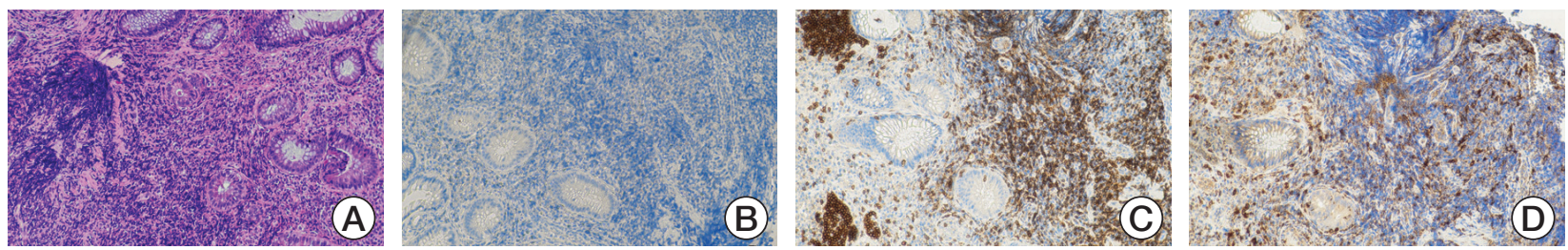

Fig. 2. (A) Active-chronic inflammation with ulceration, crypt abscess formation and goblet cell depletion (H\&E staining, $\times 5)$, (B) After rituximab therapy, CD20 cells staining was negative. (C) Immunohistochemistry with an anti-CD3 antibody demonstrated numerous intraepithelial mucosal T cells after therapy. (D) High tonaca propria and intraepithelial infiltration of macrophages $\left(C D 68^{+}\right)(B-D, \times 10)$.

dnisone $100 \mathrm{mg}$ ). He achieved complete remission and in February 2013 he began maintenance therapy with RTX (MR), at a dose of $375 \mathrm{mg} / \mathrm{m}^{2}$ every 3 months. After eight MR cycles the patient suffered a 2-month period of watery diarrhea with a frequency of 3-4 times a day, of mushy stool that occasionally contained mucus, together with periumbilical and right abdominal pain. A surveillance PET scan, performed at that time, showed an increased activity in the terminal ilium (TI) and mesenteric lymphadenopathy. An ileo-colonoscopy revealed no significant abnormality in the colon mucosa, but macroscopic inflammatory changes in the TI including an erythematous mucosa and aphthous erosions covered with fibrin. Biopsy demonstrated active nonspecific ileitis. Treatment with 5-aminosalicylates (5-ASA) induced a prompt relief of symptoms. He was treated with another six cycles of RTX for a presumed recurrence of the lymphoma. A follow-up computed tomography enterography, performed 6 months later, showed resolution of the mesenteric adenopathy but the presence of a modest hyperenhanced bowel wall thickening in the terminal ileum. In September 2017, two months after the last cycle of RTX, the patient's clinical conditions worsened. He developed bloody diarrhea, cramping abdominal pain, anemia and weight loss. Endoscopic evaluation showed a transmural involvement of the TI by an inflammatory process, with mucosal damage, deep ulceration, and edema. Histological examination revealed goblet cell depletion and active-chronic inflammation with crypt abscess formation (Fig. 2A); the lamina propria was occupied by granulation tissue with dilated, inflamed capillaries. Immunohistochemistry analysis showed a total depletion of the CD20 positive B cells in the ileal mucosa (Fig. 2B), an increased cellularity of $\mathrm{CD}^{+} \mathrm{T}$ lymphocytes in the tonaca propria and intraepithelial mucosa (Fig. 2C) and moderate excess of enlarged macrophages $\left(\mathrm{CD}^{+} 8^{+}\right)$, exclusively in the lamina propria (Fig. 2D), suggesting exacerbation of the Crohn's disease. Pathological features were in keeping with active Crohn's disease.

RTX therapy was interrupted. He was treated with budesonide and 5-ASA, and responded well. After 10 weeks the patient was asymptomatic and an endoscopic control showed slight signs of inflammation of the TI. A follow-up PET scan was negative for activity in the TI. He has remained in remission for 30 months without any adverse events. At present he is taking 5-ASA as maintenance therapy and the clinical conditions have clearly improved.

Written informed consent was obtained from the patient prior to publication of this case report and all procedures performed were in accordance with the ethical standards of the institutional research committee (IRCCS Giovanni Paolo II, Bari, Italy).

\section{Discussion}

Synchronous gastric SRC and abdominal FL is rare and there is no standard treatment strategy for patient management. A number of factors such as age, performance status, pathological features and tumor staging must be taken into consideration during decision making, in terms of which cancer to treat first and what is the optimal therapy. In our case, the patient underwent a combination of treatments, including surgical excision and chemotherapy. Subtotal gastrectomy with D2 lymphadenectomy was considered appropriate for the early-stage I-B gastric SRC treatment, while a systemic chemotherapeutic regimen was selected based on the FL bulky disease. FL is the second most common form of NHL [10]. Management is characterized by a risk-adapted therapy based on the stage of the disease and the patient's symptoms. For patients with a high tumor burden, the standard treatment option is immuno-chemotherapy. The addition of the anti-CD20 monoclonal antibody RTX to chemotherapy has yielded a higher rate of complete remission and improved survival. In addition, RTX as maintenance therapy after the induction regimen improves progression-free survival in high-tumor burden patients [11]. The use of B-cell depletion therapy with RTX has shown some success in the treatment of autoimmune diseases such as rheumatoid arthritis, by reducing the adaptive immune response against self. The elimination of B cells to treat autoimmunity also has a disadvantageous side, as it results in the depletion of regulatory $B$ cells (B-regs) that suppress inflammation [12]. Some adverse events have been reported after RTX therapy, such as cutaneous reactions, cardiomyopathies, interstitial pneumonia, infections, and progressive multifocal leukoencephalopathy. 


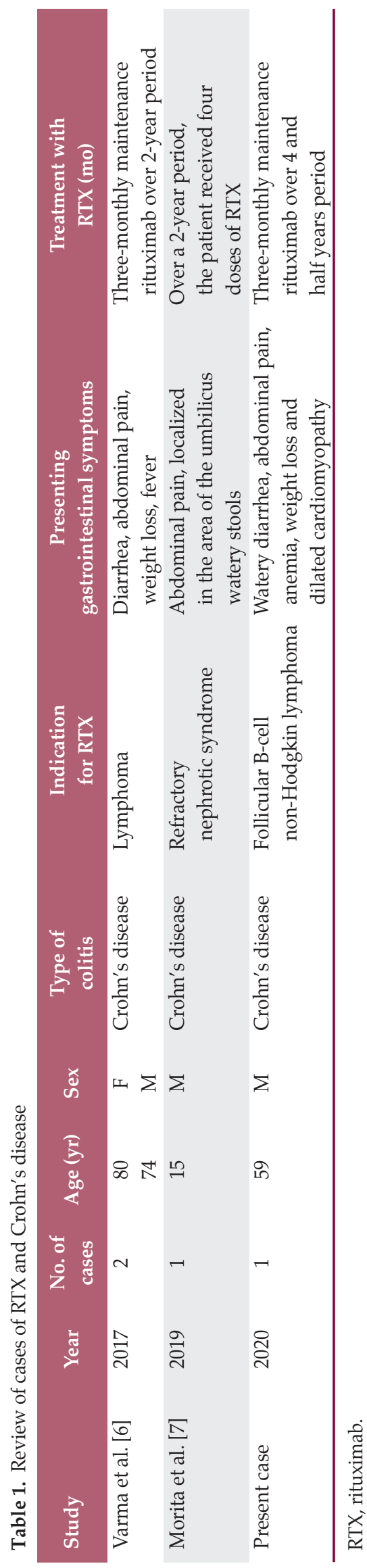

Recently, Eckmann et al. [13] described patients with RTXassociated de novo colitis, raising the hypothesis that $\mathrm{B}$ cells may play a protective role of the intestinal mucosa. There are only a few reports in the literature of the induction or exacerbation of IBD by RTX. In a patient with refractory ulcerative colitis (UC), Goetz et al. [14] reported that RTX exacerbated the disease by locking interleukin 10 (IL-10) producing B cells, suggesting an important anti-inflammatory rather than proinflammatory role of B cells in UC. To our knowledge, only three case of RTX-induced Crohn's disease have been previously reported in the literature (Table 1): two cases in elderly patients treated with 3-month MR over a 2-year period and one case of a 15-year-old boy with refractory nephrotic syndrome receiving 4 doses of RTX over a 2-year period. Compared with the cases reported previously, our patient was treated with a three-monthly MR for a longer period, fourteen doses in four and half years. At the time, the increased uptake at the mesenteric lymph nodes and at TI level, detected by the PET scan performed after eight MR cycles, suggested lymphoma recurrence and the incorrect assessment induced to continue treatment with RTX whereas, most likely, the finding was correlated with a Crohn's disease in initial phase, a rare and unexpecteted complicance. In particular this case demonstrates the importance of being alert to the possibility of Crohn's disease in presence of abdominal pain, weight loss, and diarrhea during RTX treatment. In the present report, the histopathological and immunohistochemical analysis suggests that exacerbation of Crohn's disease may be related to the total depletion of CD20 positive B cells in the intestinal mucosa and high tonaca propria and intraepithelial infiltration of the $\mathrm{T}$ cells $\left(\mathrm{CD}^{+}\right)$and macrophages $\left(\mathrm{CD} 68^{+}\right)$. B and T cells have been involved in the pathogenesis of IBD. It has been suggested that $B$ cells may not have a proinflammatory role in IBD; indeed, they may have a protective effect, reducing inflammation by producing the anti-inflammatory cytokine IL-10. Most studies performed in IBD experimental models suggest that B-regs suppress mucosal inflammation, either by secreting cytokines such as IL-10 or by interacting with T cells. Inhibition of B-T cell interaction caused by B-cell depletion may lead to T-regulatory cell dysfunction, and the subsequent activation of Th1 and Th17 cells. As a consequence, Crohn's disease could develop due to a dysregulated mucosal immune response [15]. This immune dysregulation of the gastrointestinal tract may potentially lead to Crohn's disease as a secondary complication in susceptible RTX patients. Nevertheless, the fact remains that RTX is an effective treatment for B-cell lymphoma.

In this case, we propose that Crohn's disease was caused by an abnormal dysfunction of mucosal immunity that resulted in a disturbed intestinal balance secondary to the use of RTX. Therefore, inflammatory bowel disease should be considered if severe abdominal symptoms with weight 
loss are observed following RTX administration.

\section{Conflicts of Interest}

Conflict of interest relevant to this article was not reported.

\section{Acknowledgments}

This study has been supported by Ricerca corrente 2019 .

\section{References}

1. Torres J, Mehandru S, Colombel JF, Peyrin-Biroulet L. Crohn's disease. Lancet. 2017;389:1741-55.

2. Edwards JC, Cambridge G. Sustained improvement in rheumatoid arthritis following a protocol designed to deplete $B$ lymphocytes. Rheumatology (Oxford). 2001;40:205-11.

3. Pierpont TM, Limper CB, Richards KL. Past, present, and future of rituximab: the world's first oncology monoclonal antibody therapy. Front Oncol. 2018;8:163.

4. Barreiro Alonso E, Alvarez Alvarez A, Tojo Gonzalez R, de la Coba Ortiz C. Rituximab-associated colitis. Gastroenterol Hepatol. 2019;42:251-2.

5. Mallepally N, Abu-Sbeih H, Ahmed O, Chen E, Shafi MA, Neelapu SS, et al. Clinical features of rituximab-associated gastrointestinal toxicities. Am J Clin Oncol. 2019;42:539-45.

6. Varma P, Falconer J, Aga A, Prince HM, Pianko S. Rituximabinduced Crohn's disease. Scand J Gastroenterol. 2017;52:606-8.

7. Morita K, Shibano T, Maekawa K, Hattori M, Hida N, Nakamura $\mathrm{S}$, et al. Crohn's disease following rituximab treatment in a patient with refractory nephrotic syndrome. CEN Case Rep. 2019;8:55-60.

8. Swerdlow SH, Campo E, Pileri SA, Harris NL, Stein H, Siebert R, et al. The 2016 revision of the World Health Organization classification of lymphoid neoplasms. Blood. 2016;127:2375-90.
9. Machlowska J, Puculek M, Sitarz M, Terlecki P, Maciejewski R, Sitarz R. State of the art for gastric signet ring cell carcinoma: from classification, prognosis, and genomic characteristics to specified treatments. Cancer Manag Res. 2019;11:2151-61.

10. Dada R. Diagnosis and management of follicular lymphoma: a comprehensive review. Eur J Haematol. 2019;103:152-63.

11. Buske C, Hutchings M, Ladetto M, Goede V, Mey U, Soubeyran $\mathrm{P}$, et al. ESMO Consensus Conference on malignant lymphoma: general perspectives and recommendations for the clinical management of the elderly patient with malignant lymphoma. Ann Oncol. 2018;29:544-62.

12. Mauri C, Menon M. Human regulatory B cells in health and disease: therapeutic potential. J Clin Invest. 2017;127:772-9.

13. Eckmann JD, Chedid V, Quinn KP, Bonthu N, Nehra V, Raffals LE. De novo colitis associated with rituximab in 21 patients at a tertiary center. Clin Gastroenterol Hepatol. 2020;18:252-3.

14. Goetz M, Atreya R, Ghalibafian M, Galle PR, Neurath MF. Exacerbation of ulcerative colitis after rituximab salvage therapy. Inflamm Bowel Dis. 2007;13:1365-8.

15. Xu XR, Liu CQ, Feng BS, Liu ZJ. Dysregulation of mucosal immune response in pathogenesis of inflammatory bowel disease. World J Gastroenterol. 2014;20:3255-64. 